\title{
Discrete-time Explicit Model Predictive Control for Gauge-Looper Inte- grated System in Hot Strip Finishing Mill
}

\author{
Zhaozhun Zhong ${ }^{1, *}$, Hongjing Zheng ${ }^{2}$ and Pengjie Qi ${ }^{1}$ \\ ${ }^{1}$ School of Iron and Steel Soochow University, Suzhou City, Jiangsu Province, 215021, China \\ ${ }^{2}$ School of Computer Engineering, Suzhou Vocational University, Suzhou City, Jiangsu Province, 215104, China
}

\begin{abstract}
The development of an innovative discrete-time EMPC (Explicit Model Predictive Control) strategy based on multi-parametric quadratic program is traced for the gauge-looper integrated control in HSFM (Hot Strip Finishing Mill). Controller design is based on the linearized model of the integration of downstream AGC (Automatic Gauge Control) system and upstream looper-tension system. The proposed EMPC strategy gives an explicit piecewise affine control law which greatly reduces the on-line computational burden. Discrete-time EMPC achieves better performance by coordination control and is more suitable for implementation on PLC (Programmable Logic Controller) compared with conventional controllers. Experiment results verify the effectiveness of the proposed strategy.
\end{abstract}

Keywords: Explicit model predictive control, hot strip finishing mill, gauge-looper integrated system.

\section{INTRODUCTION}

In the hot strip mill process of the iron and steel industry, heated slabs (about $250 \mathrm{~mm}$ thick and $10 \mathrm{~m}$ long) are rolled to steel pieces (about $30-35 \mathrm{~mm}$ thick and $70 \mathrm{~m}$ long) on the reversing roughing mills before entering the downstream HSFM. The HSFM process, which usually consists of six or seven close coupled finishing mills, makes further reduction of thickness by consecutive rolling and produces steel strips of required thickness and width (about $0.8-20 \mathrm{~mm}$ thick and $200-2000 \mathrm{~m}$ long) [1]. As for the steel strip product, specifications such as surface and dimensional quality, mechanical properties and stable mill operation should be satisfied. Since HSFM is the final sizing process, expensive mechanical equipments and modern control strategies are necessary to guarantee the quality of the product.

The thickness property of the steel strip is guaranteed by AGC of the hydraulic screw down system. ASR (Automatic Speed Regulator) is used to control the rolling speed of each mill to smooth the threading of the strip. During strip threading, mass flow unbalance (which affects the strip tension and stable mill operation) is frequently encountered due to disturbances. Therefore, between each pair of rolling stands, a looper system is equipped to maintain upward pressure on the strip and keep the strip tension at a desired value during operation [2]. The physical structure of conventional gaugelooper control strategy is shown in Fig. (1). It was experienced that, looper and tension control is the key to the strip dimensional quality and successful mill operation. For the last three decades, many control schemes have been proposed in the gauge-looper system: conventional PID
(Proportional Integral Derivative), Hœ, decoupling, ILQ (Inverse Linear Quadratic) for looper and tension control $[3,4]$ and conventional PI (Proportional Integral), adaptive, $\mathrm{H} \infty$, robust multivariable for AGC $[5,6]$. Conventionally, loopertension system and AGC system are controlled independently which ignores the mutual interactions among them. For example, the roll gap adjustment of AGC system causes mass flow and strip tension deviations, and the strip tension deviations also affect the rolling force and strip exit thickness inversely. Therefore, improvement would be possible by better coordination control based on an integrated system model.

Alone the line of this research trend, some multivariable control strategies have been proposed to include the gauge model in the looper-tension controller design [7]. However, the systematic coordination among gauge, strip tension and looper angle is still a remaining problem. To deal with this problem, MPC strategy, which has been proposed for the looper-tension control alone [8-10], is regarded as the one with significant potential. On one hand, MPC strategy is attractive to both practitioners and academics thanks to its ability to perform on-line optimization and constraint handling. On the other hand, traditional MPC suffers from the problem associated with computational burden due to the receding horizon policy and on-line optimization, which limits its application in slow and/or small problems. Since HSFM is a typical quick process, traditional MPC is not quite adequate. Therefore, a newly developed strategy known as EMPC (Explicit MPC), which moves all the computations of MPC off-line to reduce the on-line computational burden, is adopted for the control problem of HSFM.

Closely related downstream stand AGC system and upstream looper-tension system are unioned to form the gaugelooper integrated control system. Linear state space model of 


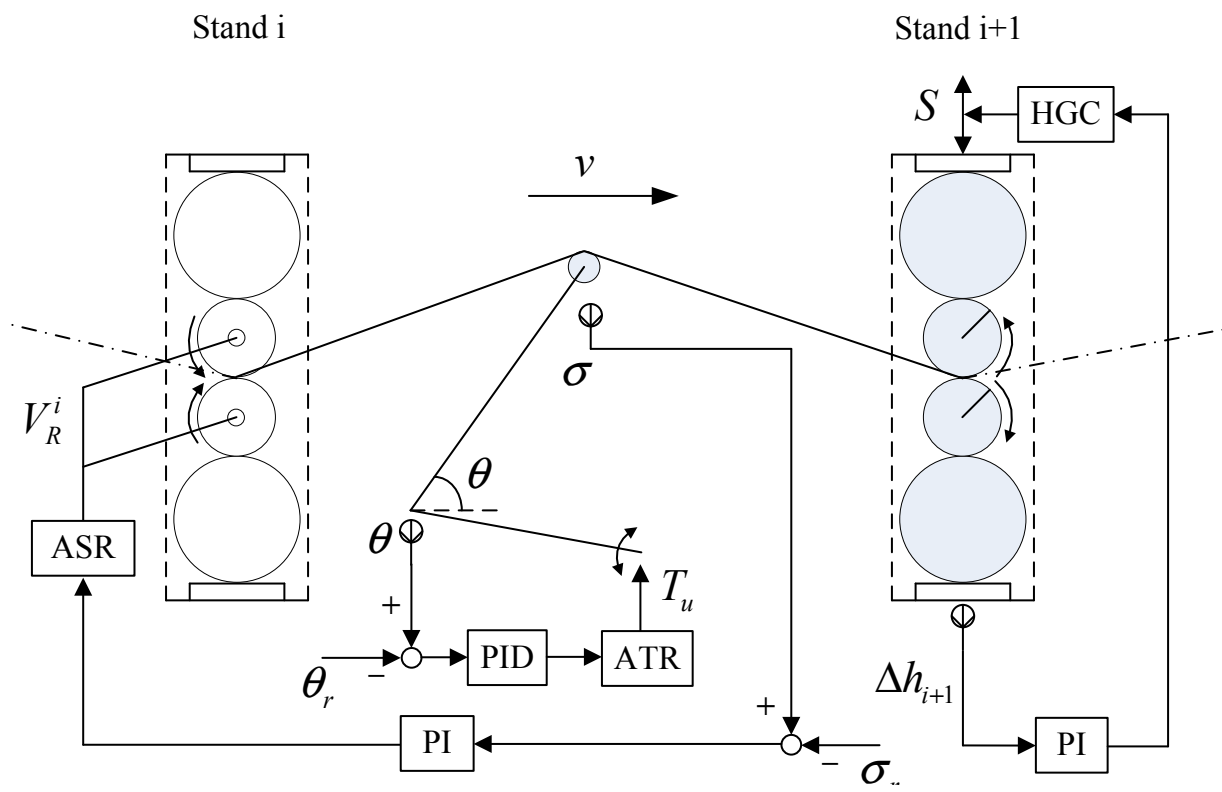

Fig. (1). Structure of conventional control strategy.

the integrated system are derived from nonlinear physical model by linear approximation about the operating point. The linear state space model is then discretized with realtime sampling interval [11]. Based on the discrete-time state space model, discrete-time EMPC is proposed to control the integrated system coordinately concerning the constraints. Instead of on-line optimization, EMPC strategy uses MPQP (Multi-Parametric Quadratic Program) to solve the optimization problem off-line. To be specific, MPQP divides the state space into critical polyhedral regions, and for each region, determines the linear gain and offset which produces the optimal control action. As a result, the solution of the EMPC optimization problem is an explicit piecewise affine function of the state which can be computed off-line [12]. The on-line computation of the EMPC optimization problem is simplified as a function evaluation, which reduces the on-line computational burden significantly and is more suitable for implementation on PLC.

The rest of the paper is organized as follows: A brief introduction of process model is given in Section 2. Proposed EMPC strategy using MPQP is discussed in Section 3. The effectiveness of the proposed EMPC strategy is verified by means of experiment in Section 4. Conclusions are given in Section 5.

\section{PROCESS MODEL}

An overview of the gauge-looper integrated system is given in this section to illustrate the background of the control problem. Plant nonlinear models of the deformation zone (shown in Fig. 2) and the interstand geometry (shown in Fig. 3) are introduced according to the physical dynamics. Linear models are derived from plant nonlinear models by approximate linearization, which will be used for controller design. The nomenclature is given in Table $\mathbf{1}$.

\subsection{Stand Model}

For the plastic deformation in the roll gap as shown in Fig. (2), the rolling force is expressed as:

$$
P=\sqrt{R_{W}^{i}\left(H_{i}-h_{i}\right)} W\left[K Q-\frac{\sigma_{f}^{i}+\sigma_{b}^{i}}{2}\right]
$$

where $h_{i}$ is the strip entry thickness, $H_{i}$ is the strip exit thickness, $\sigma_{b}^{i}$ is the strip backward tension, $\sigma_{f}^{i}$ is the strip forward tension and the rest are constant values as shown in Table 1.

The strip exit thickness is the sum of roll gap and mill stretch:

$$
h_{i}=S_{i}+\frac{P}{M_{i}}
$$

Linear model can be derived from nonlinear rolling model by approximate linearization around the set-up values. Strip exit thickness deviation of stand $i+1$ can be expressed as:

$\Delta h_{i+1}=\Delta S+\frac{\frac{\partial P}{\partial H_{i+1}} \Delta H_{i+1}+\frac{\partial P}{\partial h_{i+1}} \Delta h_{i+1}+\frac{\partial P}{\partial \sigma_{b}^{i+1}} \Delta \sigma+\Delta P_{w}}{M_{i}}$

which yields

$$
\Delta \stackrel{\mathrm{g}}{h_{i+1}}=\frac{M_{i}}{M_{i}+M_{s}} \Delta \stackrel{\mathrm{g}}{S}+\frac{M_{\sigma}}{M_{i}+M_{s}} \Delta \stackrel{\mathrm{g}}{\sigma}+\frac{M_{s}}{M_{i}+M_{s}} w_{H}+\frac{1}{M_{i}+M_{s}} w_{P}
$$

where $M_{\sigma}=\frac{\partial P}{\partial \sigma_{b}^{i+1}}, M_{s}=\frac{\partial P}{\partial H_{i+1}}=-\frac{\partial P}{\partial h_{i+1}}, w_{H}=\Delta \stackrel{g}{H_{i+1}}, \quad w_{P}=\Delta \stackrel{g}{P_{w}}$, $M_{i}$ is the mill modulus and $\Delta P_{w}$ is the unmodelled rolling force disturbance. 


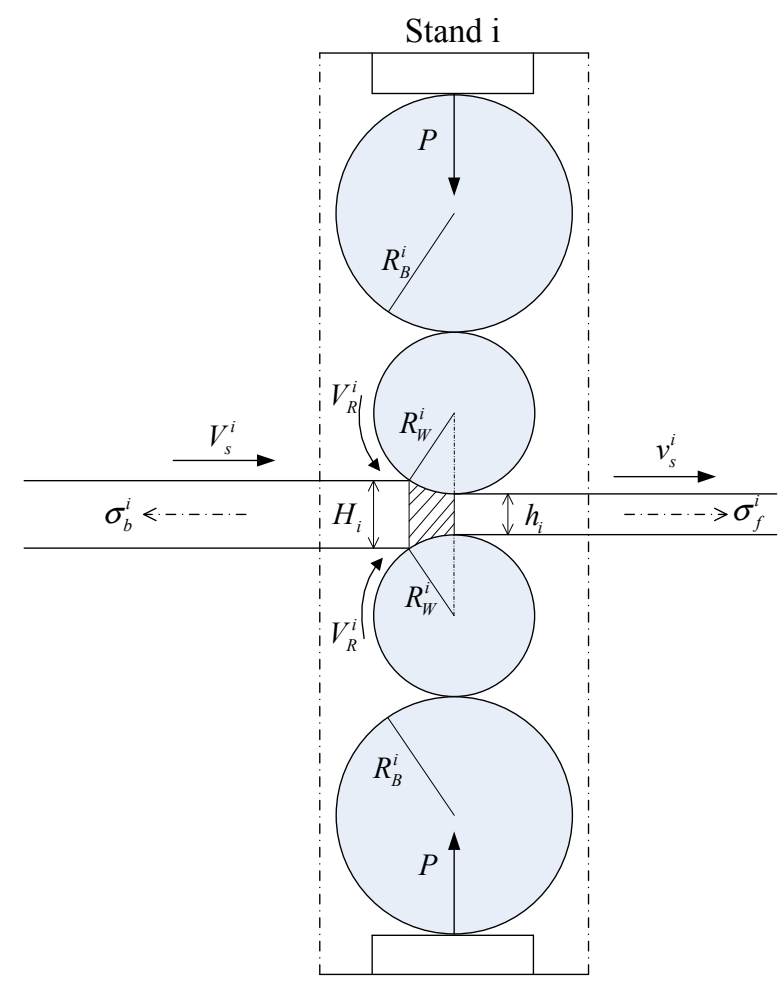

Fig. (2). Deformation zone.

Table 1. Nomenclature.

\begin{tabular}{|c|c|}
\hline Symbol & Quantity \\
\hline \hline$W$ & strip width \\
\hline$K$ & constrained yield stress \\
\hline$Q$ & correcting coefficient taking into account the geometrical aspects \\
\hline$M_{L}$ & looper mass \\
\hline$\rho$ & steel density \\
\hline$g$ & gravitational constant \\
\hline
\end{tabular}

\subsection{Strip speed} by:

The strip exit speed $v_{s}^{i}$ leaving the stand $i$ is expressed

$v_{s}^{i}=\left(1+S_{f}^{i}\right) V_{R}^{i}$

where $V_{R}^{i}(t)$ is work roll speed of stand $i . S_{f}^{i}$ is the forward slip between the work roll surface and the strip which is determined by:

$S_{f}^{i}=\frac{R_{W}^{i}}{h_{i}}\left(\gamma_{i}\right)^{2}$,

where $\gamma_{i}$ is the sliding neutral angle of the deformation zone which can be calculated as:
$\gamma_{i}=\sqrt{\frac{h_{i}}{R_{W}^{i}}} \tan \left[\begin{array}{l}\frac{1}{2} \arctan \sqrt{\frac{\varepsilon_{i}}{1-\varepsilon_{i}}}+\frac{\pi}{8} \ln \\ \left(1-\varepsilon_{i}\right) \sqrt{\frac{h_{i}}{R_{W}^{i}}}+\frac{1}{2} \sqrt{\frac{h_{i}}{R_{W}^{i}}}\left(\frac{\sigma_{f}^{i}}{K}-\frac{\sigma_{b}^{i}}{K}\right)\end{array}\right]$

$\varepsilon_{i}=\frac{H_{i}-h_{i}}{H_{i}}$.

It's obvious that, $\gamma_{i}$ is determined by strip exit thickness $h_{i}$ and strip tensions ( $\sigma_{b}^{i}$ and $\sigma_{f}^{i}$ ). The strip entry speed $V_{s}^{i}$ can be evaluated from the mass flow balance equation $v_{s}^{i} h_{i}=V_{s}^{i} H_{i}$, that is 


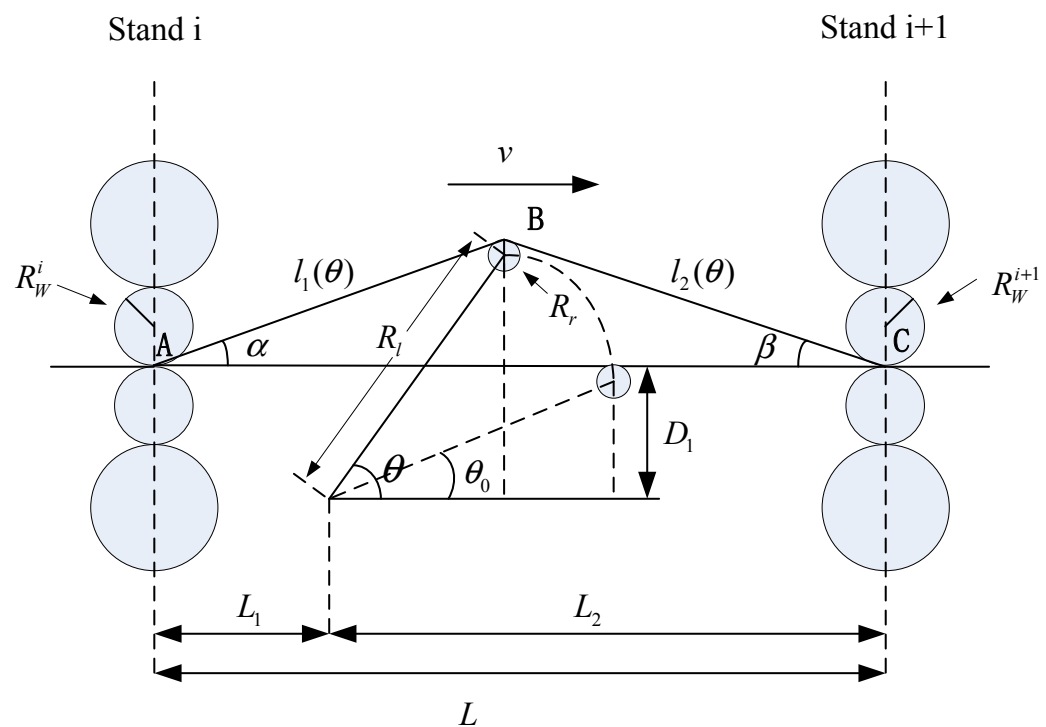

Fig. (3). Looper and interstand geometry.

$V_{s}^{i}=\frac{h_{i}}{H_{i}}\left(1+S_{f}^{i}\right) V_{R}^{i} \triangleq\left(1-S_{b}^{i}\right) V_{R}^{i}$

where $S_{b}^{i}$ is the backward slip which is also influenced by strip tensions and exit thickness.

\subsection{Tension Dynamics}

During strip threading, interstand strip tension $\sigma(t)$ (between stands $i$ and $i+1$ ) works as the forward tension $\sigma_{f}^{i}$ of stand $i$ and the backward tension $\sigma_{b}^{i+1}$ of stand $i+1$ simultaneously. It's evaluated by the amount of strip stretch between the stands:

$\sigma(t)=E\left[\frac{L^{\prime}(\theta)-(L+\xi(t))}{L+\xi(t)}\right]$ for $L^{\prime}(\theta)>(L+\xi(t))$

where $E$ is the Young's modulus of the strip, $\theta$ is the looper angle, $L$ is the interstand length. $L^{\prime}(\theta)$ is the geometric looper length between stands which can be calculated by (see Fig. 3):

$L^{\prime}(\theta)=l_{1}(\theta)+l_{2}(\theta)$

$l_{1}(\theta)=\sqrt{\left(L_{1}+R_{l} \cos \theta\right)^{2}+\left(R_{l} \sin \theta+R_{r}-D_{1}\right)^{2}}$

$l_{2}(\theta)=\sqrt{\left(L_{2}-R_{l} \cos \theta\right)^{2}+\left(R_{l} \sin \theta+R_{r}-D_{1}\right)^{2}}$

$L+\xi(t)$ is the accumulated material length which is caused by the speed difference between the coupling stands and whose derivative is given by:

$\dot{\xi}(t)=v_{s}^{i}(t)-V_{s}^{i+1}(t)+w_{\xi}(t)$, where $v_{s}^{i}(t)$ is the strip speed leaving stand $i$ and $V_{s}^{i+1}(t)$ the strip speed entering stand $i+1$ which are given by (3) and (5) respectively, $w_{\xi}(t)$ represents unmodelled perturbations caused by the fast action of AGC system in the case of disturbances.

Since $\xi(t)$ is rather small compared with $L$, it's ignored in the denominator of (6). As a result, the derivative of $\sigma(t)$ is given by:

$$
\begin{aligned}
\dot{\sigma}(t) & =\frac{E}{L}\left[\frac{d}{d t} L^{\prime}(\theta)-\dot{\xi}(t)\right] \\
& =\frac{E}{L}\left[R_{l}[\sin (\theta+\beta)-\sin (\theta-\alpha)] \dot{\theta}(t)-\left(v(t)+w_{\xi}(t)\right)\right]
\end{aligned}
$$

where upstream strip angle $\alpha$ and downstream strip angle $\beta$ are evaluated

$\alpha=\tan ^{-1}\left[\left(R_{l} \sin \theta-D_{1}+R_{r}\right) /\left(L_{1}+R_{l} \cos \theta\right)\right]$ and $\beta=\tan ^{-1}\left[\left(R_{l} \sin \theta-D_{1}+R_{r}\right) /\left(L_{2}-R_{l} \cos \theta\right)\right]$ respectively.

Therefore, interstand strip tension deviation for linear model can be expressed as:

$$
\begin{aligned}
\dot{\Delta \sigma}= & \frac{E}{L} F_{3}(\theta) \Delta \omega-\frac{E}{L}\left(\frac{\partial S_{f}^{i}}{\partial \sigma_{f}^{i}} V_{R}^{i}-\frac{h_{i+1}}{H_{i+1}} \frac{\partial S_{f}^{i+1}}{\partial \sigma_{b}^{i+1}} V_{R}^{i+1}\right) \Delta \sigma \\
& -\frac{E}{L}\left(1+S_{f}^{i}\right) \Delta V_{R}^{i}+\frac{E}{L} \frac{1}{H_{i+1}}\left(1+S_{f}^{i+1}+h_{i+1} \frac{\partial S_{f}^{i+1}}{\partial h_{i+1}}\right) V_{R}^{i+1} \Delta h_{i+1} \\
& -\frac{E}{L} \frac{h_{i+1}}{H_{i+1}^{2}}\left(1+S_{f}^{i+1}-H_{i+1} \frac{\partial S_{f}^{i+1}}{\partial H_{i+1}}\right) V_{R}^{i+1} \Delta H_{i+1}+w_{\xi}(t)
\end{aligned}
$$




\subsection{Looper Dynamics}

Looper dynamics can be derived by applying Newton's law of motion to the looper system, that is:

$J \ddot{\theta}(t)=T_{u}(t)-T_{\text {load }}(\theta)+w_{\omega}(t)$

where $J$ is the total inertia of the looper with respect to the pivoting point, $T_{u}(t)$ is the actuator torque on the looper. $w_{\omega}(t)$ denotes the unmodelled dynamics caused by the vicious friction phenomena of the hydraulic cylinder, the torque on the looper to bend the strip and so on. $T_{\text {load }}(\theta)$ represents the load torque on the looper which is the combination of the loads caused by strip tension $T_{\sigma}(\theta)$, strip weight $T_{s}(\theta)$ and looper weight $T_{L}(\theta)$, that is:

$T_{\text {load }}(\theta)=T_{\sigma}(\theta)+T_{s}(\theta)+T_{L}(\theta)$

where $\quad T_{\sigma}(\theta)=\sigma h_{i} W R_{l}[\sin (\theta+\beta)-\sin (\theta-\alpha)] \quad$, $T_{L}(\theta)=g M_{L} R_{G} \cos \theta$ and $T_{s}(\theta) \approx 0.5 g \rho L h_{i} W R_{l} \cos \theta$.

Looper angular speed deviation is then expressed by:

$\dot{\Delta \omega}=-\frac{1}{J} \frac{\partial T_{\text {load }}}{\partial \theta} \Delta \theta-\frac{1}{J} \frac{\partial T_{\sigma}}{\partial \sigma} \Delta \sigma+\frac{1}{J} \Delta T_{u}+\frac{1}{J} w_{\omega}(t)$

\subsection{Actuator Dynamics}

As for the actuators, they are actually based on preexisting basic controllers: the gap of the stand $S$ is regulated by HGC (Hydraulic Gap Control), the stand work roll speed $V_{R}^{i}(t)$ is controlled by ASR and the looper actuator torque $T_{u}(t)$ is controlled by ATR (Automatic Torque Regulator). They are subject to dynamics which are not negligible and can be modeled as first-order dynamics with time constants $T_{A T R}, T_{A S R}$ and $T_{H G C}$ respectively.

$\dot{S(t)}=-\frac{1}{T_{H G C}} S(t)+\frac{1}{T_{H G C}} u_{S}$

$\dot{V}_{R}^{i}(t)=-\frac{1}{T_{A S R}} V_{R}^{i}(t)+\frac{1}{T_{A S R}} u_{V}$

$\dot{T}_{u}(t)=-\frac{1}{T_{A T R}} T_{u}(t)+\frac{1}{T_{A T R}} u_{T}$

where $u_{S}, u_{V}$ and $u_{T}$ are the control inputs of $S(t), V_{R}^{i}(t)$ and $T_{u}(t)$ respectively. $T_{H G C}, T_{A S R}$ and $T_{A T R}$ are the time constants of the first-order dynamics of HGC, ASR and ATR respectively.

The variables to be summarized in the control input vector $u$ are represented by the variations with respect to their nominal values of the reference variables for HGC, ASR and ATR, that is,

$$
u=\left[\begin{array}{lll}
u_{\Delta T} & u_{\Delta V} & u_{\Delta S}
\end{array}\right]^{T}
$$

And the actuator dynamics can be rewritten as

$$
\begin{aligned}
& \Delta \Delta \dot{S}(t)=-\frac{1}{T_{H G C}} \Delta S(t)+\frac{1}{T_{H G C}} u_{\Delta S} \\
& \left\{\Delta V_{R}^{i}(t)=-\frac{1}{T_{A S R}} \Delta V_{R}^{i}(t)+\frac{1}{T_{A S R}} u_{\Delta V}\right. \\
& \Delta \dot{T_{u}}(t)=-\frac{1}{T_{A T R}} \Delta T_{u}(t)+\frac{1}{T_{A T R}} u_{\Delta T}
\end{aligned}
$$

\subsection{Linearized Process Model}

As we can see from the process model introduced in Sections 2.1-2.5, the AGC system of stand $i+1$ is closely related with the looper-tension system between stand $i$ and stand of $i+1$. A linearized mathematical model of the gaugelooper integrated system can be derived by the union of linear equations (2), (8), (10) and (11). The state variables of the linearized model are chosen as the variations of the states with respect to their nominal values:

$$
x=\left[\begin{array}{lllllllll}
\Delta I_{\theta} & \Delta \theta & \Delta \omega & \Delta I_{\sigma} & \Delta \sigma & \Delta I_{h} & \Delta T_{u} & \Delta V_{R}^{i} & \Delta h_{i+1}
\end{array}\right]^{T}
$$

where $\Delta I_{\theta}, \Delta I_{\sigma}$ and $\Delta I_{h}$ are induced integral of the system outputs $\Delta \theta, \Delta \sigma$ and $\Delta h_{i+1}$ respectively, which are used to eliminate the steady-state error.

As a result, the linear state space model of the gaugelooper integrated system is expressed as:

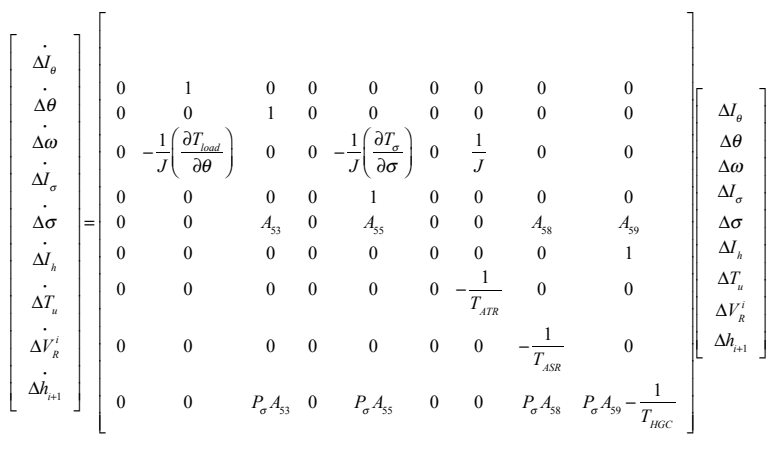


where $A_{53}=\frac{E}{L} F_{3}(\theta), \quad P_{\sigma}=\frac{M_{\sigma}}{M_{m}+M_{s}}, \quad P_{m}=\frac{M_{m}}{M_{m}+M_{s}}$, $P_{1}=\frac{1}{M_{m}+M_{s}} \quad, \quad P_{s}=\frac{M_{s}}{M_{m}+M_{s}} \quad, \quad A_{55}=-\frac{E}{L}$ $\left(\frac{\partial S_{f}^{i}}{\partial \sigma_{f}^{i}} V_{R}^{i}-\frac{h_{i+1}}{H_{i+1}} \frac{\partial S_{f}^{i+1}}{\partial \sigma_{b}^{i+1}} V_{R}^{i+1}\right), \quad A_{58}=-\frac{E}{L}\left(1+S_{f}^{i}\right), \quad A_{59}=\frac{E}{L H_{i+1}}$ $\left(1+S_{f}^{i+1}+h_{i+1} \frac{\partial S_{f}^{i+1}}{\partial h_{i+1}}\right) V_{R}^{i+1}$.

Looper angle, strip tension and strip exit thickness are chosen as the outputs of the integrated system, that is

$$
y=\left[\begin{array}{lllllllll}
0 & 1 & 0 & 0 & 0 & 0 & 0 & 0 & 0 \\
0 & 0 & 0 & 0 & 1 & 0 & 0 & 0 & 0 \\
0 & 0 & 0 & 0 & 0 & 0 & 0 & 0 & 1
\end{array}\right]\left[\begin{array}{c}
\Delta I_{\theta} \\
\Delta \theta \\
\Delta \omega \\
\Delta I_{\sigma} \\
\Delta \sigma \\
\Delta I_{h} \\
\Delta T_{u} \\
\Delta V_{R}^{i} \\
\Delta h_{i+1}
\end{array}\right]
$$

The control problem is defined as a set point regulator problem, that is, the outputs (strip exit thickness $h_{i}(t)$, looper angle $\theta(t)$ and strip tension $\sigma(t))$ should be regulated at the desired reference values.

\section{INTEGRATED CONTROL BASED ON EMPC}

\subsection{EMPC Based on MPQP: A Brief Review}

For the sake of the readers' convenience, a brief review of EMPC based on multi-parametric quadratic program, which has been applied to typical linear quadratic control for constrained systems [13], is given in this section.

Consider a discrete-time MIMO time-invariant linear system of the regular form:

$$
\left\{\begin{array}{l}
x(k+1)=A x(k)+B u(k) \\
y(k)=C x(k)
\end{array}\right.
$$

while fulfilling the constraints

$$
y_{\min } \leq y(k) \leq y_{\max }, u_{\min } \leq u(k) \leq u_{\max }
$$

at all time instants $k \geq 0$. In (14)-(15), the state vector $x(k) \in \mathbb{R}^{n}$, the input vector $u(k) \in \mathbb{R}^{p}$, and the output vector $y(k) \in \mathbb{R}^{q}, A, B$ and $C$ are matrices of proper dimension.
It's obvious that, for system (14), given the initial state $x(k)=x_{k}$, the system dynamic will be solely determined by the control input sequence $u_{k+j} \triangleq u(k+j), j \geq 0$. Therefore, MPC solves the following optimization problem:

$\min _{U \triangleq\left\{u_{k}, \cdots, u_{k+N_{u}-1}\right\}}\left\{\begin{array}{l}J(U, x(k))=x_{k+N_{p}}^{\prime} P x_{k+N_{p}} \\ +\sum_{j=0}^{N_{p}-1}\left[x_{k+j}^{\prime} Q x_{k+j}+u_{k+j}^{\prime} R u_{k+j}\right]\end{array}\right\}$

s.t.

$$
\begin{aligned}
& y_{\min } \leq y_{k+j} \leq y_{\max }, j=1, \cdots, N_{p} ; \\
& u_{\min } \leq u_{k+j} \leq u_{\max }, j=0,1, \cdots, N_{u}-1 ; \\
& x_{k+j+1}=A x_{k+j}+B u_{k+j}, j \geq 0 ; \\
& y_{k+j}=C x_{k+j}, j \geq 0 ; \\
& u_{k+j}=0, \quad N_{u} \leq j \leq N_{p}-1 .
\end{aligned}
$$

at each time $k$, where $N_{u}$ is length of the control input sequence, $N_{p} \geq N_{u}$ is the pre-defined length of the optimal window, and $x_{k+j}$ denotes the predicted state vector at time $k+j$ as the response of the control input sequence $U$ applied to system (14) starting from the initial state $x_{k}$.

The spirit of MPC is the construction of an optimal control input sequence $U^{*}=\left\{u_{k}^{*}, u_{k+1}^{*}, \cdots, u_{k+N_{u}-1}^{*}\right\}$ which minimizes the cost function $J$ in (16) with constraints (17). And it's necessary to point out that, according to the receding horizon control principle, only the first step of the control input $U^{*}$ (i.e., $u^{*}(k)$ ) is taken into the system at the time instant $k$. As for $k+1$, the optimal problem (16) will be solved once again and only the first step of the solution is taken into the system. In other words, this optimal programming will be taken over and over again along the time sequence to guarantee the stability of the closed-loop system under unmodelled dynamics, disturbances, and so on. Such a control strategy is known as receding horizon control.

The above stated MPC problem has been investigated by numerous researchers over the last few decades and a solid theoretical foundation for MPC has emerged. However, it usually has the drawback of heavy on-line computational burden. In this paper, we adopt a recently proposed EMPC strategy based on multi-parametric quadratic program which is able to move all the computations of MPC off-line [12]. And the resulting controller is a explicit piecewise affine function of the states which is suitable for the discrete-time linear model of the gauge-looper integrated system derived in Section 2.6.

By substituting $x_{k+j}=A^{j} x(k)+\sum_{m=0}^{j-1} A^{j-m-1} B u_{k+m}$, the optimal problem (16) can be rewritten in compact form as 
$V^{*}\left(x_{k}\right)=\frac{1}{2} x_{k}^{\prime} Y x_{k}+\min _{U}\left\{\frac{1}{2} U^{\prime} H U+x_{k}^{\prime} F U\right.$, s.t. $\left.G U \leq W+E x_{k}\right\}$

where $U=\left[u_{k}^{\prime}, u_{k+1}^{\prime}, \cdots, u_{k+N_{u}-1}^{\prime}\right]^{\prime}$ is the optimization vector, and $H, F, Y, G, W, E$ are easily obtained from $Q$ and $R$ in (16). As proposed in [12], the quadratic program (18) can be solved by multi-parametric quadratic program.

For the sake of convenience, (18) is transferred into the following form by setting $z \triangleq U+H^{-1} F^{\prime} x_{k}$ :

$V_{z}^{*}\left(x_{k}\right)=\min _{z} \frac{1}{2} z^{\prime} H z$

s.t. $G z \leq W+S x_{k}$

where $S \triangleq E+G H^{-1} F^{\prime}$ and

$V_{z}^{*}\left(x_{k}\right)=V^{*}\left(x_{k}\right)-\frac{1}{2} x_{k}^{\prime}\left(Y-F H^{-1} F^{\prime}\right) x_{k}$.

For the multi-parametric quadratic programming problem (19)-(20), we introduce the following result which is the key to construct a piecewise affine state-feedback control law for EMPC.

Lemma 1. [12]. For a quadratic programming problem stated in (19)-(20), let $z=z_{0}^{*}$ be the optimal solution for a given state $x_{k}^{0}$ and $\{\tilde{G}, \tilde{W}, \tilde{S}\}$ is the uniquely determined set of active constraints $\tilde{G} z_{0}^{*}=\tilde{W}+\tilde{S} x_{k}^{0}$ out of the constraints in (20). Assume that the rows of $\tilde{G}$ are linearly independent, and let $C R_{0}$ be the set of all vectors $x_{k}$ for which the combination of constraints $\{\tilde{G}, \tilde{W}, \tilde{S}\}$ is active at the optimum ( $C R_{0}$ is referred to as critical region). Then, the optimal solution $z^{*}$ of (19)-(20) is a uniquely defined affine function of $x_{k}$.

$z^{*}=H^{-1} \tilde{G}^{\prime}\left(\tilde{G} H^{-1} \tilde{G}^{\prime}\right)^{-1}\left(\tilde{W}+\tilde{S} x_{k}\right)$

over the polyhedral region $C R_{0}$ defined by

$G H^{-1} \tilde{G}^{\prime}\left(\tilde{G} H^{-1} \tilde{G}^{\prime}\right)^{-1}\left(\tilde{W}+\tilde{S} x_{k}\right) \leq W+S x_{k}^{0}$

$\left(\tilde{G} H^{-1} \tilde{G}^{\prime}\right)^{-1}\left(\tilde{W}+\tilde{S} x_{k}\right) \leq 0$

To summarize, multi-parametric quadratic programming systematically subdivides the space $X$ of parameters $x_{k}$ into critical regions ( $C R s)$. For each $C R$, the optimal solution $z^{*}$ is an affine function of $x_{k}$. Once the critical region $C R_{0}$ has been defined, the rest of the space $C R^{\text {rest }} \triangleq X \backslash C R_{0}$ can be explored and new critical regions will be generated by an iterative algorithm which partition $C R^{\text {rest }}$ recursively.
In order to introduce the iterative algorithm, a few methods should be demonstrated at first.

(i) For a polyhedral set $X=\left\{x_{k}: T x_{k} \leq v\right\}$, a good choice for $x_{k}^{0} \in X$ to start solving the multi-parametric quadratic program problem (19)-(20) will be the center of the largest ball contained in $X$ for which feasible $z$ exists, which is determined by solving the linear program (LP).

$$
\begin{array}{ll}
\max _{x_{k}, z, \varepsilon} & \varepsilon \\
\text { s.t. } & T^{j} x_{k}+\varepsilon\left\|T^{j}\right\| \leq v^{j} \\
& G z-S x_{k} \leq W
\end{array}
$$

(ii) Let $X_{1} \subseteq \mathbb{R}^{n}$ be a polyhedron, and $C R_{0} \triangleq\left\{x \in X_{1}: T_{0} x \leq v_{0}\right\} \quad$ a polyhedron subset of $X_{1}$, $C R_{0} \neq \varnothing$, let

$R_{i}=\left\{\begin{array}{cc}x \in X_{1}: & T_{0}^{i} x>v_{0}^{i} \\ & T_{0}^{j} x \leq v_{0}^{j}, \forall j<i\end{array}\right\}, i=1, \cdots, m$,

where $m=\operatorname{dim}\left(v_{0}\right)$. Then, $\left\{C R_{0}, R_{1}, \cdots, R_{m}\right\}$ is a partition of $X_{1}$.

(iii) For a polyhedral set $X$ and the multi-parametric quadratic program problem (19)-(20), define an operator $\operatorname{Part}(X)$

1) Determine $x_{k}^{0} \in X$ by solving the LP (24), if corresponding $\varepsilon \leq 0$ then exit (there is no critical region in $X$ );

2) For $x_{k}^{0} \in X$, calculate the optimal solution $z_{0}^{*}$ of the QP (19)-(20);

3) Determine the set of active constraints $\{\tilde{G}, \tilde{W}, \tilde{S}\}$ for $x_{k}^{0}, z_{0}^{*}$, if the rows of $\tilde{G}$ is not linearly independent, take the largest subset of linear independent rows and redefine $\{\tilde{G}, \tilde{W}, \tilde{S}\}$

4) Determine $z^{*}=f\left(x_{k}\right)$ from (21) for critical region $C R_{0}$ (22)-(23);

5) Define and partition $C R^{r e s t}=X \backslash C R_{0}$ as in (25);

6) For each new sub-region $R_{i}$, execute $\operatorname{Part}\left(R_{i}\right)$;

7) End procedure.

Based on the above discussions, the off-line mp-QP solver are outlined briefly as follows

Algorithm 1:

a) Let $X$ be the set of parameters/states;

b) Execute $\operatorname{part}(X)$; 


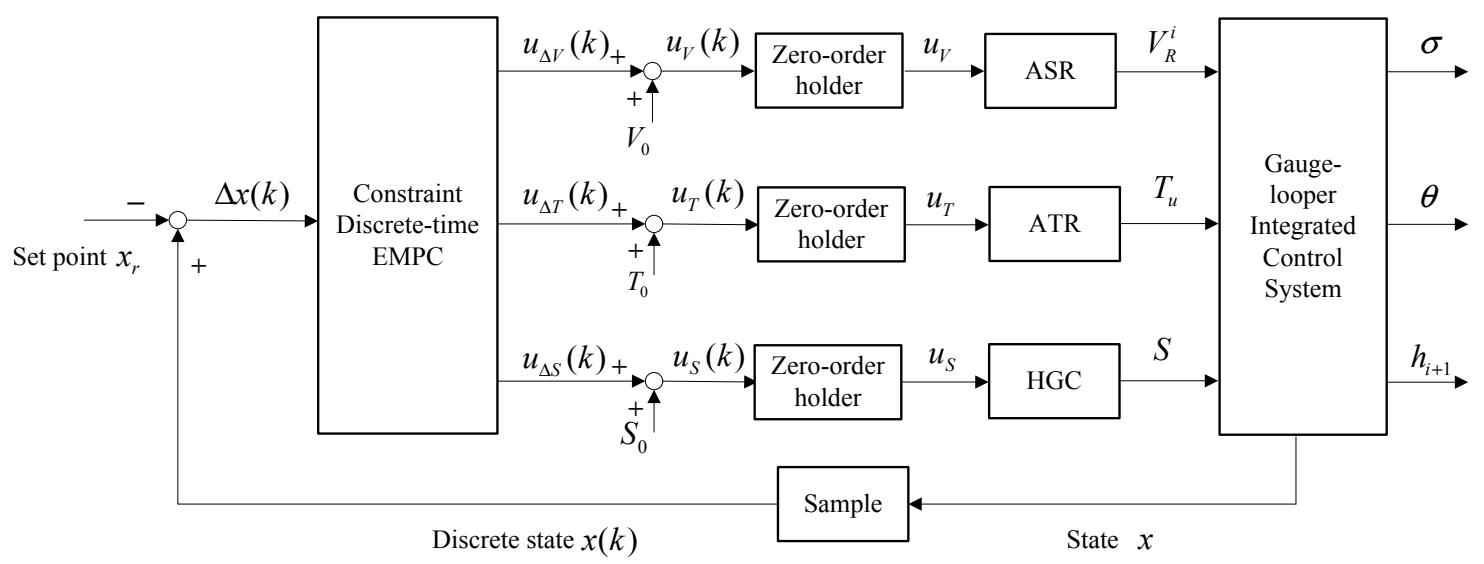

Fig. (4). Discrete-time EMPC control structure.

c) Unite all the regions where $z^{*}\left(x_{k}\right)$ is the same and whose union is a convex set to reduces the total number of regions;

d) End.

As a result, $X$ are divided into critical regions, and in each region, the optimal solution $z^{*}\left(x_{k}\right)$ is an affine function of $x_{k}$ (i.e. $z^{*}\left(x_{k}\right)$ is piecewise affine). And the application of Algorithm 1 to the DMPC problem (16)-(17) is straight forward, as a result, the optimal control sequence $U^{*}$ is a piecewise affine function of $x_{k}$, so does $u_{k}^{*}$ with the form

$u_{k}^{*}=F^{j} x_{k}+g^{j}$, if $T^{j} x_{k} \leq v^{j}$

where $j=1, \cdots, N_{m p c}$ and $N_{m p c}$ is the number of regions.

\subsection{EMPC Controller Design}

For the sake of clarity and simplicity, we calculate the approximately linearized model of the gauge-looper integrated system. Thus we have:

$\left\{\begin{array}{l}\dot{x}(t)=\bar{A} x(t)+\bar{B} u(t)+\bar{D} w(t) \\ y(t)=C x(t)\end{array}\right.$

where corresponding vectors and matrices are defined in Section 2.6.

In order to apply the proposed EMPC strategy, (27) is discretized with $T=0.025 \mathrm{~s}$ (sampling interval of the real-time control system) which yields:

$$
\left\{\begin{array}{l}
x(k+1)=A x(k)+B u(k)+D w(k) \\
y(k)=C x(k)
\end{array}\right.
$$

Based on the discrete-time linear model (28), piecewise affine EMPC controllers can be designed according to the standard procedure discussed in Section 3.1 [14].
The overall control structure is shown in Fig. (4) and the control parameters will be given in Section 4 .

\section{EXPERIMENT RESULTS}

The proposed EMPC strategy is evaluated by experiment taken in a HSFM process. ASR of Stand 3, ATR of Looper 4 and AGC of Stand 5 are taken from a seven-stand finishing mill process to form an integrated gauge-looper system. The looper angle reference $\theta_{r}$ is set as $20^{\circ}$ and the strip tension reference $\sigma_{r}$ is set as $8 \mathrm{MPa}$.

As far as the characteristics of the integrated system are concerned, we set predictive horizon as $N_{p}=16$, control horizon as $N_{u}=10$. Weight matrices are selected by analysis of step response and frequency-singular value plots:

$Q=\left[\begin{array}{ccc}1 & 0 & 0 \\ 0 & 0.5 & 0 \\ 0 & 0 & 1.5\end{array}\right]$ and $R=\left[\begin{array}{ccc}1 & 0 & 0 \\ 0 & 40 & 0 \\ 0 & 0 & 25\end{array}\right]$.

And the constraints are taken as: $\left|u_{\Delta T}\right| \leq 4,\left|u_{\Delta V}\right| \leq 2$, $\left|u_{\Delta S}\right| \leq 10,|\Delta \theta| \leq 2,|\Delta \sigma| \leq 4$ and $\left|\Delta h_{i+1}\right| \leq 30$.

Experiment results of the proposed EMPC strategy as well as the conventional PID controllers are given in Figs. (5-7). In the case of EMPC, the looper is more stable, strip tension tracks the reference more exactly and the thickness deviation is reduced. For example, EMPC reduces the peakto-peak tension fluctuation by $30 \%$ compared with conventional PID. To sum up, EMPC achieves better disturbance rejection by systematical coordination among gauge, strip tension and looper angle, which can easily be tuned by adjusting the weight matrices. 

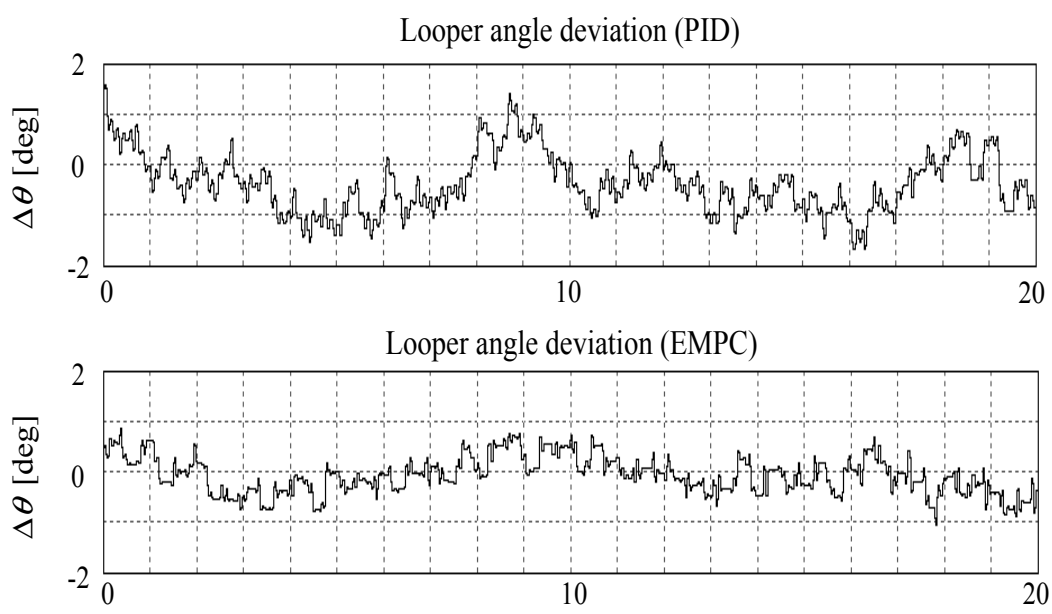

Fig. (5). Plant data of the looper angle deviation.
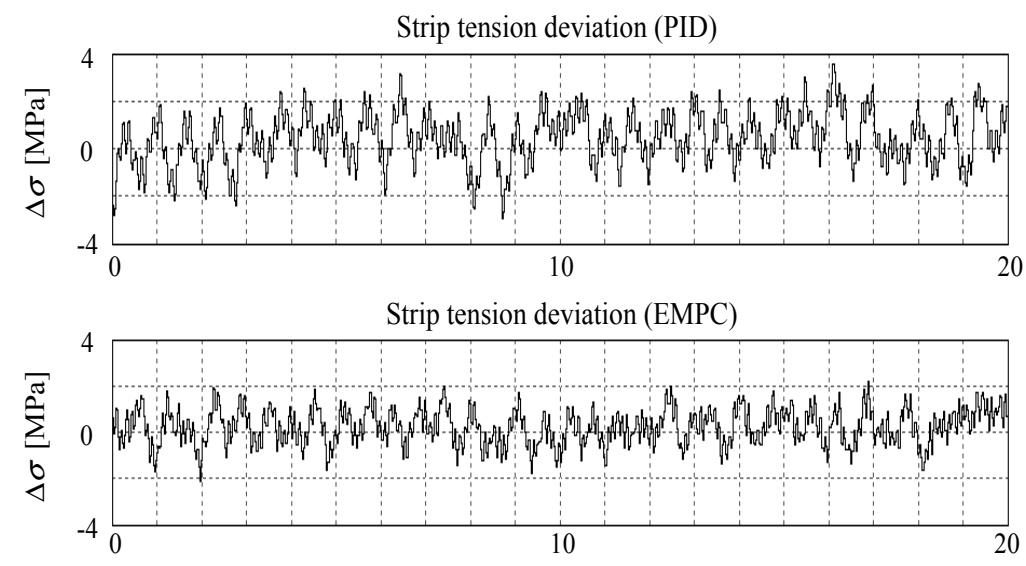

Fig. (6). Plant data of the strip tension deviation.
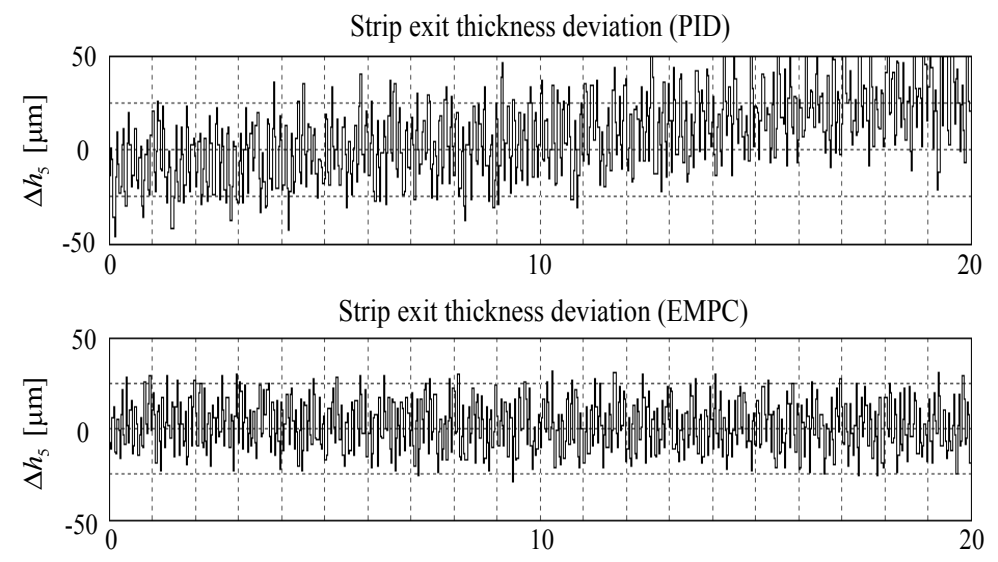

Fig. (7). Plant data of the strip thickness deviation.

\section{CONCLUSION}

An innovative EMPC strategy is proposed for gaugelooper integrated control in HSFM. The interactions between the AGC system and the looper-tension system are reduced by systematical coordination control of the integrated system based on EMPC. EMPC strategy gives an explicit piecewise affine control law based on MPQP which greatly reduces the on-line computational burden compared with conventional MPC strategy. Experiment results verify the effectiveness of the proposed strategy. 
Future research effort could be the development of good prediction models and the formulation of a robust EMPC algorithm which satisfies state and input constraints under parameters uncertainties and disturbances.

\section{CONFLICT OF INTEREST}

The authors confirm that this article content has no conflicts of interest.

\section{ACKNOWLEDGEMENTS}

This work was partially supported by National Natural Science Foundation of China (61304095 \& 61403254), Natural Science Foundation of Jiangsu Province (BK20130317), Shanghai Natural Science Foundation of China (13ZR1428500), Jiangsu Planned Projects for Postdoctoral Research Funds (1302103B) and Suzhou Science and Technology Program (SGZ2013135).

\section{REFERENCES}

[1] I.S. Choi, J.A. Rossiter, and P.J. Fleming, "Looper and tension control in hot rolling mills: A survey," Journal of Process Control, vol. 17, no. 6, pp. 509-521, 2007.

[2] J.S. Farrokh, and J. Liu, "Design of a self-adaptive fuzzy tension controller for tandem rolling," IEEE Transactions Industrial Electron., vol. 52, no. 5, pp. 1428-1438, 2005.

[3] Z. Z. Zhong, and J.C. Wang, "Looper-tension almost disturbance decoupling control for hot strip finishing mill based on feedback linearization," IEEE Transactions on Industrial Electronics, vol. 58 , no. 8, pp. 3668-3679, 2011.
[4] F.A. Cuzzola, "A multivariable and multi-objective approach for the control of hot-strip mills," Transactions ASME, vol. 128, pp. 856-868, 2006

[5] G. Hearns, and M.J. Grimble, "Robust multivariable control for hot strip mills," ISIJ International, vol.40, no.10, pp. 995-1002, 2000.

[6] T. Ohta, and Y. Washikita, "Adaptive control for the head-end strip gauge using recursive least squares at hot strip mill," In: Proceedings on IEEE International Conference on Control Applications, Munich, Germany, 2006, pp. 1831-1836.

[7] M. Okada, Y. Iwasaki, K. Murayama, A. Urano, A. Kawano, and H. Shiomi, "Optimal control system for hot strip finishing mill," In: Proceedings of the $35^{\text {th }}$ IEEE Conference on Decision and Control, Kobe, Japan, 1996, pp. 1236-1241.

[8] J. S. Choi, A. Rossiter, and P. Fleming, "Effectiveness of MPC algorithms for hot rolling mills in the presence of disturbances," In: Proceedings on American Control Conferences on Control Applications, New York, USA, 2007, pp. 4118-4123.

[9] J. Schuurmans, and T. Jones, "Control of mass flow in a hot strip mill using model predictive control," In: Proceedings IEEE International Conference on Control Applications, Glasgow, U.K. 2002, pp. 379-384

[10] A. Kojima, and N. Morooka, "Start up control of a hot strip mill tension/looper system: an approach based on model predictive control," In: Proceedings $17^{\text {th }}$ World Congress IFAC, Seoul, 2008, pp. 1651-1656.

[11] P. Russu, "Controlling complex dynamics in a protected area discrete-time model," Discrete Dynamics in Nature and Society, vol. 2012, Article ID 432319, 13 pages, 2012. Doi:10.1155/2012/432319

[12] A. Bemporad, M. Morari, V. Dua, and E. N. Pistikopoulos, "The explicit linear quadratic regulator for constrained systems," Automatica, vol. 38, pp. 3-20, 2002.

[13] Kojima, and M. Morari, "LQ control for constrained continuoustime systems," Automatica, vol. 40, pp. 1143-1155, 2004.

[14] F. Borrelli, "Constrained optimal control of linear and hybrid systems," Lecture Notes in Control and Information Sciences, Springer: Breton, Heidelberg, p. 290, 2003 